Kansas State University Libraries

New Prairie Press

Conference on Applied Statistics in Agriculture 2007 - 19th Annual Conference Proceedings

\title{
Editor's Preface and Table of Contents
}

John E. Boyer Jr.

Follow this and additional works at: https://newprairiepress.org/agstatconference

Part of the Agriculture Commons, and the Applied Statistics Commons

\section{c) $($ ) $@ \Theta$}

This work is licensed under a Creative Commons Attribution-Noncommercial-No Derivative Works 4.0 License.

\section{Recommended Citation}

Boyer, John E. Jr. (2007). "Editor's Preface and Table of Contents," Conference on Applied Statistics in Agriculture. https://doi.org/10.4148/2475-7772.1104

This is brought to you for free and open access by the Conferences at New Prairie Press. It has been accepted for inclusion in Conference on Applied Statistics in Agriculture by an authorized administrator of New Prairie Press. For more information, please contact cads@k-state.edu. 


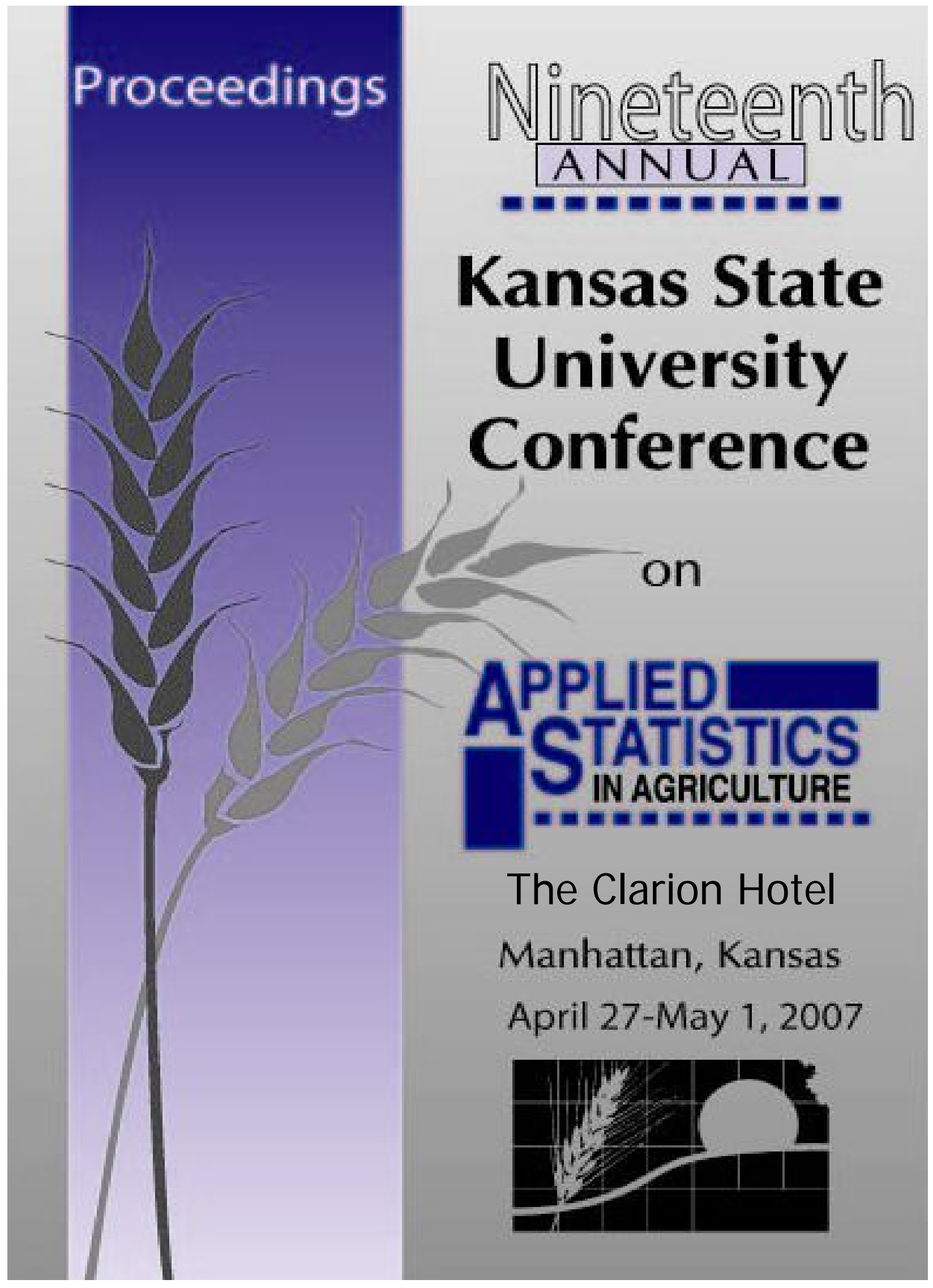




\section{Preface}

These proceedings contain papers presented in the nineteenth annual Kansas State University Conference on Applied Statistics in Agriculture, held in Manhattan, Kansas, April 27-May 1, 2007. The purpose of the conference is to provide a forum for discussion on the application of statistics to problems in agricultural sciences. Papers and posters presented at the conference and included in the proceedings are intended to provide information to both the statistician and agricultural researcher on current issues of agricultural and general statistical interest.

The keynote speaker was Dr. R.W. Doerge, with Purdue University, West Lafayette, Indiana. On April 28, 2007, Dr. Doerge presented a workshop titled "Introduction to Quantitative Trait Locus (QTL) Mapping in Experimental Populations: Past and Present”.

Each contributed paper was refereed before being accepted for publication in these proceedings. The papers were accepted for publication based on their interest, relevance, innovation and application to the agricultural sciences and statistics.

Thanks goes out to the following referees: Bruce Craig, Philip Dixon, Stuart Gardner, Ed Gbur, Carla Goad, Jennifer Huckett, Dallas Johnson, Matt Kramer, Steve Lund, Bruce Mackey, Ben Mallinix, George Milliken, Anne Parkhurst, Bill Price, John Stevens, Caryn Thompson, Jessica Thomson, Bryan Vinyard, Kevin Wright and Man Yu Yum; to the staff members for their help in assembling the proceedings; and finally to our conference sponsors: American Statistical Association, Eli Lilly \& Company, Pfizer Inc, Pioneer Hi Bred-a DuPont Business and the USDA-ARS for their continued support.

John E. Boyer, Jr., Ph. D.

Proceedings Editor 


\section{Table of Contents}

\section{Tridimensional Regression}

Kendra Schmid, University of Nebraska-Lincoln

David Marx, University of Nebraska-Lincoln

Ashok Samal, University of Nebraska-Lincoln

Alternative Estimation Techniques for Assessing Probability of Frost Damage in

Subalpine Fir Trees

William J. Price, University of Idaho

Bahman Shaffi, University of Idaho

Danny L. Barney, University of Idaho

Using the Cumlative-Size Mechanistic Model for Analyzing Insect Data

T.I. Matis, Texas Tech

J.H. Matis, Texas A\&M

G.J. Michels Jr., Texas Agriculture Experiment Station-Bushland

M.N. Parajulee, Texas Agriculture Experiment Station-Lubbock

Specifications of a Prototype Software System for Developing Variable-Rate Treatment Prescriptions for Use in Precision Agriculture

Kevin S. McCarter, Louisiana State University-Baton Rouge

Eugene Burris, Louisiana State University AgCenter-St. Joseph

George A. Milliken, Kansas State University

Ernest L. Clawson, Louisiana State University AgCenter-St. Joseph

Hoi Yee Wong, Louisiana State University-Baton Rouge

Jeffrey L. Willers, USDA/ARS Mississippi State University

Selection of Blocked Two-Level Fractional Factorial Designs for Agricultural Experiments

Weiming Ke, South Dakota State University

Cuirong Ren, South Dakota State University

Huitian Lu, South Dakota State University

Adjusting Population Estimates for Genotyping Error in Non-Invasive DNA-Based

Mark-Recapture Experiments

Shannon M. Knapp, Purdue University

Bruce A. Craig, Purdue University

Tolerance Intervals for Gene Flow Rates from Transgenic to Non-Transgenic Wheat and Corn Using a Logistic Regression Model with Random Location Effects

Samuel Broderick, Colorado State University

Phillip Chapman, Colorado State University

Patrick Byrne, Colorado State University

Todd Gaines, Colorado State University 
Using Nonlinear Fixed and Mixed Models with Switch Functions to Allow for Hormesis in Growth of Escherichia Coli

C. Tu, University of Nebraska-Lincoln

A.M. Parkhurst, University of Nebraska-Lincoln

L.M. Durso, University of Nebraska-Lincoln

R.W. Hutkins, University of Nebraska-Lincoln

Using a Nonlinear Crossed Random Effects Model with Three-Way Treatment Structure for Describing Circadian Patterns of Serum Prolactin Concentrations in Heat Stressed Holsteins

M. Zhou, University of Nebraska-Lincoln

A.M. Parkhurst, University of Nebraska-Lincoln

B.C. Pollard, University of Arizona-Tucson

R.J. Collier, University of Arizona-Tucson

Sample Size Determination in Animal Health Studies

Zhanglin Cui, Eli Lilly \& Company

Alan G. Zimmermann, Eli Lilly \& Company

Daniel H. Mowrey, Eli Lilly \& Company

Spectral Procedures Enhance the Analysis of Three Agricultural Time Series David Meek, USDA-ARS-MWA - National Soil Tilth Laboratory, Ames, IA

J. Prueger, USDA-ARS-MWA - National Soil Tilth Laboratory, Ames, IA M. Tomer, USDA-ARS-MWA - National Soil Tilth Laboratory, Ames, IA

R. Malone, USDA-ARS-MWA - National Soil Tilth Laboratory, Ames, IA

2007 Listing of Conference Attendees 


\section{LIST OF ATTENDEES}

An, Lingling - Purdue University

Bargen, Jason - Pfizer Inc

Bauer, Karin - Midwest Research Institute

Biven, Laura - US Department of Agriculture

Blankenship, Erin - Univ. of Nebraska-Lincoln

Boyer, John - Kansas State University

Boykin, Debbie - USDA/ARS

Brennan, Meghan - University of Florida

Bryson, Lawrence - Pfizer Animal Health

Bsharat, Rebhi - Kansas State University

Burch, Brent - Northern Arizona University

Camp, Mary - USDA/ARS

Chapman, Jessica - Iowa State University

Chapman, Phillip - Colorado State University

Cheng, Lili - Kansas State University

Christman, Mary - University of Florida

Cochrane, Chun Yen - Kansas State University

Cornelius, Paul - University of Kentucky

Craig, Bruce - Purdue University

Cui, Lin - Eli Lilly \& Company

Dey, Rajarshi - Kansas State Univesity

Doerge, Rebecca - Purdue University

Douglass, Larry - University of Maryland

Duke, Sara - USDA/ARS

Durham, Susan - Utah State University

Feng, Lizhi - Pioneer Hi Bred, a DuPont Business

Fernandez, George - University of Nevada-Reno

Ferry, Nancy - DuPont Crop Protection

Gbur, Edward - University of Arkansas

Goad, Carla - Oklahoma State University

Goodwin, Roger - USDA/Nat'l Agricultural Stats Svcs

Guo, Shengjie - University of Nebraska-Lincoln

Higgins, James - Kansas State University

Hinds, Mark - Pioneer Hi Bred, a DuPont Business

Hobbs, Jonathan - Iowa State University

Hoffman, Wherly - Eli Lilly \& Company

Hollinger, Amy - Southeast Missouri State University

Hooks, Tisha - University of Nebraska-Lincoln

House, John - St. Luke's Hospital

Huang, Qiwen - University of Nebraska-Lincoln

Jayawardhana, Ananda - Pittsburg State University

Jiang, Pingping - University of Missouri

Johnson, Dallas - Kansas State University

Jung, Yoonsung - Kansas State University

Kastens, Jude - Kansas University

Ke, Weiming - South Dakota State University
Khamis, Imad - Southeast Missouri State University Kiernan, Kathleen - SAS Institute

Kim, Sungwook - Pittsburg State University

Knight, Christopher - Novus International Inc

Koehler, Kenneth - Iowa State University

Kramer, Matt - USDA/ARS

Kratzer, Dal - ESCI

Lamsal, Sunil - Southeast Missouri State University

Leone, Erin - University of Maryland

Leyva-Estrada, Norma - Iowa State University

Liu, Grace - Pioneer Hi Bred, a DuPont Business

Loughin, Thomas - Kansas State University

Loughin, Marie - Milliken Associates Inc.

Lu, Huitian - South Dakota State University

Luo, Lang

MacKinnon, April - Milliken Associates Inc.

Macchiavelli, Raul - University of Puerto Rico

Mackey, Bruce - USDA/ARS

Marx, David - University of Nebraska-Lincoln

McCarter, Kevin - Louisiana State University

Meek, David - USDA/ARS

Meyer, Corrie - Southeast Missouri State University

Milliken, George - Kansas State University

Min, Jun - Kansas State University

Mo, Hua - Pioneer Hi Bred, a DuPont Business

Mohanty, Radha - Monsanto Company

Mowrey, Daniel - Eli Lilly \& Company

Mullinix, Benjamin - UGA Tifton Campus

Neill, Jim - Kansas State University

Nemeth, Margaret - Monsanto Company

Njuho, Peter - Kansas State University

Ogunyinka, Ebenezer - Kansas State University

Palmquist, Deb - USDA/ARS

Parkhurst, Anne - University of Nebraska-Lincoln

Payne, Rob - Degussa Corporation

Phillips, Greg - USDA

Price, William - University of Idaho

Qiao, Meihua - Eli Lilly \& Company

Quinlan, Michelle - University of Nebraska-Lincoln

Recknor, Justin - Iowa State University

Remund, Kirk - Monsanto Company

Ren, Cuirong - South Dakota State University

Richardson, Troy - Kansas State University

Ridpath, Harold - USDA/APHIS

Rodriquez, Jorge - Kansas State University

Roozeboom, Kraig - Kansas State University 


\section{LIST OF ATTENDEES}

van Santen, Edzard - Auburn University

Schroeppel, Stephen - Southeast Missouri State Univ.

Senne, Lane - PRA International

Shafii, Bahman - University of Idaho

Shrestha, Smriti - Kansas State University

Simon Stephen - Childrens Mercy Hospital

Singh, Pradeep - Southeast Missouri State University

Smith, Christina - Kansas State University

Song, Jiuzhou - University of Maryland

St. Pierre, Normand - Ohio State University

Staudhammer, Christina - University of Florida

Steadman, Greg

Stevens, John - Purdue University

Stroup, Walt - University of Nebraska-Lincoln

Sun, Zhiwei - Kansas State University

Sweeney, Diane - Schering-Plough Animal Health

Tang, Zhongwen - Kansas State University

Tanner, James - Tanner Consulting

Taylor, Veronica - FDA

Thomson, Jessica - USDA

Tolos, Siti - Kansas State University

Travnicek, Daryl - University of Nebraska-Lincoln
Van Vleck, Dale - USDA/ARS

VanLeeuwen, Dawn - New Mexico State University

Vinyard, Bryan - USDA/ARS

Wang, Dong - Iowa State University

Wang, Yi - University of Nebrask-Lincoln

West, Mark - USDA/ARS

Whitehand, Linda - USDA/ARS/WRRC

Wilson, Samuel - Kansas State University

Wright, Deanne - Pioneer Hi Bred, a DuPont Business

Wright, Kevin - Pioneer Hi Bred, a DuPont Business

Wroughton, Jacqueline - University of Nebraska-Lincoln

Xie, Lin - Kansas State University

Yang, Celeste - Kansas State University

Yang, Xiao - Southeast Missouri State University

Yeater, Kathy - USDA/ARS

Young, Linda - University of Florida

Zeng, Beiyan - Monsanto Company

Zhang, Kurt - Kansas State University

Zhang, Wuyan

Zhao, Huiyan - Monsanto Company

Zhou, Meijian - University of Nebraska-Lincoln 